\title{
La Formación Profesional Básica y su contribución al desarollo de competencias para el reenganche educativo y la inserción laboral: percepción del alumnado
}

\author{
M. Carmen Sarceda-Gorgoso \\ Eva M. Barreira-Cerqueiras \\ Universidad de Santiago de Compostela. España. \\ carmen.sarceda@usc.es \\ evamaria.barreira@usc.es
}

Recibido: $7 / 7 / 2020$

Aceptado: 30/9/2020

Publicado: 5/7/2021

\section{Resumen}

La Ley para la mejora de la calidad educativa da luz a las enseñanzas de FP Básica como medida dirigida al alumnado en riesgo de fracaso escolar o que haya fracasado de facto, con una doble finalidad: facilitar su permanencia en el sistema educativo y ofrecer mayores posibilidades para su desarrollo personal y profesional acreditando una cualificación de nivel 1. En este trabajo, de corte cuantitativo y con el cuestionario como instrumento de recogida de información, se indaga sobre la percepción que el alumnado de FP Básica de la comunidad autónoma de Galicia tiene sobre su desarrollo competencial profesional. Participan en él un total de 148 estudiantes de los cursos primero y segundo, hombres en su mayor parte, y que están matriculados en un ciclo de la familia profesional de electricidad y electrónica. Sus respuestas permiten afirmar que, en general, consideran tener un desarrollo competencial medio o incluso medio-alto, y que la variable género influye en aquellas competencias de carácter más transversal, mientras que la familia profesional contribuye en mayor o menor medida al desarrollo de aquellas competencias más vinculadas con la profesionalización del individuo.

Palabras clave: desarrollo de competencias; educación y formación profesional; Formación Profesional Básica; vulnerabilidad

Resum. La formació professional bàsica i la seva contribució al desenvolupament de competències per al reenganxament educatiu i la inserció laboral: percepció de l'alumnat

La Llei per a la millora de la qualitat educativa dona llum als ensenyaments d'FP bàsica com a mesura dirigida a l'alumnat que pateix risc de fracàs escolar o que hagi fracassat de facto, amb una doble finalitat: facilitar-ne la permanència en el sistema educatiu i oferir més possibilitats per al seu desenvolupament personal i professional acreditant una qualificació de nivell 1. En aquest treball, de tall quantitatiu i amb el qüestionari com a instrument de recollida d'informació, s'indaga sobre la percepció que l'alumnat d'FP bàsica de la comunitat autònoma de Galícia té sobre el seu desenvolupament competencial professional. Hi participen un total de 148 estudiants dels cursos primer i segon, la major part dels quals són homes que están matriculats en un cicle de la família professional d'electri- 
citat i electrònica. Les seves respostes permeten afirmar que, en general, consideren que tenen un desenvolupament competencial mitjà o fins i tot mitjà-alt, i que la variable gènere influeix en aquelles competències de caràcter més transversal, mentre que la família professional contribueix en una mesura més o menys gran a promoure el desenvolupament d'aquelles competències més vinculades amb la professionalització de l'individu.

Paraules clau: desenvolupament de competències; educació i formació professional; formació professional bàsica; vulnerabilitat

Abstract. Basic vocational training and its contribution to the development of competences for educational re-engagement and labor insertion: Student perception

Basic vocational education and training (VET) was established under the Spanish Law for the Improvement of Educational Quality as a measure aimed at students at risk of school failure or who have failed, with a dual purpose: to facilitate their permanence in the educational system and offer more opportunities for personal and professional development by accrediting a level 1 qualification. This work, which is of a quantitative nature and uses a questionnaire as an instrument for collecting information, investigates the perception that Basic VET students of the autonomous region of Galicia have of their professional competence development. A total of 148 students (1st and 2 nd year) participated in the study, the majority of whom were men in the basic degree cycle of the electricity and electronics professional field. Their responses confirm that, in general, they consider that they have an intermediate or even intermediate-high competence level, and that the gender variable influences competences of a more transversal nature, while the professional field contributes to a greater or lesser extent to the development of competences more closely related to the professionalization of the individual.

Keywords: skills development; vocational education and training; basic vocational training; vulnerability

\section{Sumario}

$\begin{aligned} \text { 1. Introducción } & \text { 4. Discusión y conclusiones } \\ \text { 2. Método } & \text { Referencias bibliográficas }\end{aligned}$

3. Resultados

\section{Introducción}

La Ley Orgánica 5/2002 define la formación profesional como la comprensión de un «conjunto de acciones formativas que capacitan para el desempeño cualificado de las diversas profesiones, el acceso al empleo y la participación activa en la vida social, cultural y económica» (p. 22440). Más recientemente, la Ley Orgánica 8/2013 (LOMCE) introduce la Formación Profesional Básica (en adelante FP Básica) como una enseñanza de formación profesional que comparte los fines y aspectos específicos de las enseñanzas profesionalizadoras. El Real Decreto 127/2014 establece, además, que su objeto es la adquisición de las competencias profesionales, personales y sociales, y de las competencias del aprendizaje permanente a lo largo de la vida. 
Esta finalidad, sin duda incuestionable, resulta especialmente importante si se atiende al colectivo que cursa FP Básica: alumnado entre 15 y 17 años que, habiendo cursado tercero de ESO o, excepcionalmente, segundo, esté en riesgo de fracaso escolar o haya fracasado de facto. Es, por tanto, un colectivo especialmente vulnerable que precisa de una adecuada respuesta del sistema educativo a su derecho a una educación inclusiva que le permita alcanzar los resultados de aprendizaje vinculados a las competencias profesionales del título y, así, favorecer su incorporación al mercado de trabajo. No se puede olvidar que, según datos de Eurostat correspondientes a julio de 2019, España tiene el dudoso honor de liderar la tasa de abandono educativo con el nivel más alto de los 28 países de la Unión Europea (un 17,9\%), con valores similares a los de Malta (un 17,5\%) o Rumanía (un 16,4\%).

Por otra parte, y en lo que respecta a la inserción laboral, los datos ofrecidos por la Encuesta de Población Activa correspondientes al primer trimestre de 2020 muestran una situación altamente preocupante con relación a este colectivo. La tasa de paro entre la juventud con edades comprendidas entre los 16 y los 19 años, y cuya formación se limita a la primera etapa de educación secundaria, se sitúa en un $47,5 \%$. Si introducimos la variable género, la situación se ve agravada, puesto que llega a situarse en el 50,54\% en el caso de las mujeres. No son solo muy malos números, sino que resulta especialmente alarmante su reiteración en el tiempo.

Este oscuro panorama se vuelve especialmente peligroso en situaciones límite de crisis económica, en las que se magnifican las diferencias en la empleabilidad entre jóvenes con formación y aquellos que no la tienen (Serrano y Soler, 2015). Actualmente, en España se prevé, por causa del COVID-19, una recesión estimada del $12 \%$ en el mejor escenario, siendo diversos los organismos que advierten que nuestro país va a ser de los más azotados por la pandemia, tal y como se pone de relieve en un informe de Deutsche Bank, en el que se afirma que

[...] todos los organismos oficiales y privados han rebajado estimaciones de crecimiento para este año y el que viene. Para España, las advertencias son constantes y la OCDE o el FMI nos coloca como una de las economías desarrolladas que más van a sufrir con el COVID-19, alertando del desastre. (Deutsche Bank, 2020, p. 1)

Parece evidente que los costes y la profundidad de la crisis serán enormes y, de nuevo, afectarán en mayor medida a los colectivos más desfavorecidos, tal y como ya sucedió con la crisis del 2007 y anteriormente con las de los años 1980 y 1990, que trajeron como consecuencia un escenario caracterizado por reducir las perspectivas de empleo de manera substancial y oscurecer los horizontes de futuro de modo más que notable y, por ende, unas expectativas de nivel de vida más que problemáticas (Brunet, Pizzi y Valls, 2013; Serrano y Soler, 2015), incrementando, tal y como afirman Olmos-Rueda y Mas-Torelló (2017), la situación de vulnerabilidad y riesgo de exclusión de los jóvenes que no acrediten una titulación académica básica. Desde este punto de partida 
resulta de suma importancia que el sistema educativo sea capaz de promover el desarrollo de competencias profesionalizadoras. A este respecto, asumimos el concepto de competencia definido por Tejada, entendiéndolo como «un conjunto de conocimientos, procedimientos y actitudes combinados, coordinados e integrados en el sentido que el individuo ha de 'saber hacer' y 'saber estar' para el ejercicio profesional» (Tejada, 1999, p. 27).

Complementariamente, una cuestión que no se puede olvidar hace referencia a las repercusiones negativas que tiene para los jóvenes abandonar el sistema educativo sin haber completado la enseñanza secundaria, y que se traducen no solo en un amplio abanico de dificultades a nivel de inserción laboral (Sarceda-Gorgoso, Santos-González, y Sanjuán Roca, 2017; Serrano y Soler, 2015) y en una menor propensión a participar en acciones de formación continua (EURYDICE y CEDEFOP, 2014), sino que desde una perspectiva global trae como consecuencia un mayor nivel de vulnerabilidad en el colectivo afectado (OECD, 2016a, 2016b).

Así las cosas, la formación de estos jóvenes (no solo en términos de nivel educativo alcanzado, sino, sobre todo, en lo que tiene que ver con el nivel de competencia obtenido) se convierte en la última oportunidad para una inserción laboral efectiva que les permita superar el negro futuro que se les avecina. En este sentido, tal y como señalan Olmos-Rueda y Mas-Torelló (2017), el proceso de adquisición y desarrollo de las competencias está orientado a la búsqueda de mayores niveles de empleabilidad.

Aunque la FP Básica es relativamente reciente y, por ende, son escasos los estudios que la abordan, de la revisión de la literatura sobre FP Básica se constata la diversidad en las temáticas abordadas. Así, Sarceda-Gorgoso et al. (2017) realizan un diagnóstico de la FPB como medida para paliar el fracaso escolar, mientras que González-González y Cutanda-López (2020) establecen las condiciones organizativas necesarias para que los programas de FP Básica se conviertan en eficaces medidas de reenganche educativo. Fernández-García, García y García (2019), por su parte, se centran en el papel de la FP Básica como alternativa para atender a las necesidades educativas de los jóvenes en riesgo social. También las TIC son objeto de atención, tanto en lo que concierne a la competencia digital del alumnado de FP Básica (Moreno-Guerrero, Fuentes y López, 2019) como a las implicaciones del contexto social, económico y cultural de este alumnado para la utilización de dichos dispositivos (Moreno-Guerrero, López, Pozo y Fuentes, 2019). Atendiendo a la inserción laboral, Sarceda-Gorgoso, Santos-González, Barreira-Cerqueiras y Sanjuán-Roca (2019) exploran el potencial de estas enseñanzas con relación al trinomio diversidad, éxito educativo y empleabilidad, mientras que Olmos-Rueda y Mas-Torelló (2017) abordan la perspectiva de tutores y de empresas sobre el desarrollo de las competencias básicas de empleabilidad en el marco de los programas de FP Básica. Por su parte, Negri y Leiva (2019) analizan el papel de sus docentes en la inserción sociolaboral de jóvenes con diversidad funcional intelectual.

Estas investigaciones evidencian el valor de la FP Básica como medida niveladora de desigualdades. Si además consideramos que la investigación 
sobre el tema - a pesar de su evidente importancia — es todavía muy escasa, trabajos como el que se presenta se dotan de un valor añadido.

\section{Método}

El estudio se caractiza por una metodología de corte cuantitativo, más concretamente adopta una perspectiva estadística no experimental. El tratamiento y análisis ha sido efectuado a través del programa estadístico SPSS 25.0 para Windows.

\subsection{Objetivos de investigación}

1) Conocer la autopercepción del alumnado de FPB sobre el desarrollo de sus competencias profesionales.

2) Identificar variables que influyen en esta autopercepción.

\subsection{Instrumento}

El instrumento utilizado para la recogida de información ha sido un cuestionario elaborado ad hoc, conformado por 5 dimensiones (datos generales, competencias y contenidos transversales, tutoría, FCT y competencia digital) que se responden — exceptuando la primera — en una escala de medida tipo Likert de 5 grados. El instrumento fue sometido a juicio de expertos, quienes revisaron los ítems según su pertinencia. Una vez realizada la aplicación piloto a 30 estudiantes, la fiabilidad para el conjunto del cuestionario se sitúa en $\alpha=0,978$, valor que indica una muy buena consistencia interna.

La aplicación definitiva tuvo carácter presencial y se llevó a cabo en el aula en un momento acordado con el tutor o la tutora del grupo (presente en el momento de la aplicación), de manera que se interfiriera lo menos posible en la actividad ordinaria. Previamente se había solicitado un consentimiento informado por parte de los padres o de los tutores legales de los participantes, dado que en su mayoría eran menores de edad.

\subsection{Participantes}

La muestra participante, de carácter incidental, la componen 148 estudiantes de los cursos primero y segundo de FP Básica de la comunidad autónoma de Galicia, con una media de edad de 16,51 años.

De ella, el 74,3\% son hombres, mientras que las mujeres representan el $25,7 \%$. Así mismo, del total, un 35,8\% cuenta con 17 años, seguido de un $33,8 \%$ que tiene 16 años.

Atendiendo a la familia profesional del ciclo que están realizando, se percibe un claro predominio de la rama de Electricidad y Electrónica (un 31,1\%), Administración y Gestión (un 19,6\%), Automoción (un 14,9\%) y Agraria (un $14,2 \%)$. Con mucha menor presencia se encuentran las familias de Hostelería 
y Turismo (un 6,8\%) y de Informática y Comunicaciones (un 3,4\%). Es necesario destacar que en todas estas familias, tanto las que poseen mayor presencia como menor presencia, se observa una clara preeminencia de los hombres frente a las mujeres, a excepción de Automoción, en la cual no existe representación femenina.

La mayoría de los encuestados (un 97,3\%) ha accedido a la FP Básica a través de la ESO, y al 72,1\% de ellos no les hubiera gustado seguir cursando estas enseñanzas. Así mismo, aunque a un $81 \%$ del total de la muestra le gusta el ciclo que está realizando, se observa un alto porcentaje de encuestados, un $57,1 \%$, al que le hubiera gustado realizar un ciclo de FP Básica de una familia profesional distinta a la actual.

En cuanto a su futuro profesional, un $44,6 \%$ de los encuestados indica que le interesaría tener un trabajo relacionado con su ciclo actual (a un $23 \%$ le interesaría "bastante» y a un 21,6\% le interesaría "mucho»), mientras que el $32,4 \%$ de la muestra señala que le interesaría "nada» (un 10,1\%) o "poco» (un $22,3 \%)$ ejercer una profesión vinculada con el ciclo actual. En un lugar intermedio encontramos a un $23 \%$ de la muestra, que señala que le interesaría «algo» este aspecto.

Igualmente, se preguntó a los alumnos acerca de la continuación de estudios tras finalizar el ciclo formativo que están cursando, a lo cual un $80,7 \%$ de la muestra señala que sí ha pensado continuar estudiando, frente a un $19,3 \%$ que indica una respuesta negativa.

\section{Resultados}

Como primer objetivo de este trabajo, interesaba conocer la autopercepción del alumnado de FP Básica sobre el desarrollo de sus competencias profesionales. Para ello se realiza un análisis descriptivo de las valoraciones realizadas por los participantes acerca del nivel de desarrollo que estos poseen de una serie de competencias, gracias al ciclo de FP Básica que están cursando. Las competencias analizadas son afines al desarrollo de la profesión y al mundo del trabajo, pero su adquisición también contribuye a las posibilidades de continuación de estudios.

Conviene observar que en la mayor parte de las variables seleccionadas (tabla 1) existen una serie de valores perdidos que es preciso tener en cuenta (Durán, 2005; Montenegro, Oh y Chesnut, 2015) a fin de evitar sesgos y aportar un mayor grado de confiabilidad en las conclusiones. A pesar de que no se trata de una pérdida excesiva de casos para cada variable, realizamos un análisis de valores perdidos ${ }^{1}$ utilizando la regresión y concluyendo que no existe prácticamente ninguna diferencia entre los valores de los estadísticos con respuesta (media y desviación típica) y los obtenidos con la aplicación de esta prueba (media de regresión y desviación típica estimada).

1. El propio programa estadístico empleado nos señala que en el análisis realizado no hay variables con el $5 \%$ o más de sus valores perdidos. 
Tabla 1. Valores y puntuaciones de estadísticos descriptivos para las competencias analizadas (V18 a V23 y V28 a V31)

\begin{tabular}{|c|c|c|c|c|c|}
\hline & $\mathbf{N}$ & $x$ & $\mathbf{S}$ & $c_{v}$ & $g_{1}$ \\
\hline Desarrollar competencias del título profesional (V18) & 146 & 3,52 & 1,045 & $29,7 \%$ & $-0,514$ \\
\hline Comprender el sector productivo correspondiente (V19) & 146 & 3,34 & 1,078 & $32,3 \%$ & $-0,269$ \\
\hline Aprender por sí mismo (V20) & 145 & 3,77 & ,888 & $23,5 \%$ & $-0,501$ \\
\hline Aprender a trabajar en equipo (V21) & 147 & 3,89 & 1,048 & $27 \%$ & $-0,794$ \\
\hline Conocer y prevenir riesgos laborales (V22) & 141 & 3,79 & 1,052 & $27,7 \%$ & $-0,846$ \\
\hline Desarrollar el espíritu emprendedor (V23) & 148 & 3,59 & 1,130 & $31,5 \%$ & $-0,525$ \\
\hline $\begin{array}{l}\text { Desarrollar competencias relacionadas con la educación } \\
\text { cívica (V28) }\end{array}$ & 146 & 3,10 & 1,104 & $35,6 \%$ & $-0,348$ \\
\hline $\begin{array}{l}\text { Desarrollar competencias relacionadas con la comunica- } \\
\text { ción audiovisual (V29) }\end{array}$ & 144 & 3,03 & 1,161 & $38,3 \%$ & $-0,096$ \\
\hline Desarrollar competencias relacionadas con las TIC (V30) & 146 & 3,23 & 1,022 & $31,6 \%$ & $-0,310$ \\
\hline $\begin{array}{l}\text { Prepararme para la continuación en el sistema educativo } \\
\text { (V31) }\end{array}$ & 148 & 3,52 & 1,091 & $31 \%$ & $-0,674$ \\
\hline
\end{tabular}

Fuente: elaboración propia.

Así pues, retomando el análisis descriptivo, hay que señalar que la puntuación otorgada al conjunto de competencias indica que la percepción de los encuestados en cuanto a su nivel de desarrollo se sitúa, grosso modo, en un nivel intermedio. Esto se confirma en base al coeficiente de asimetría $\left(g_{1}\right)$ de cada variable - negativos todos ellos-, lo que parece indicar que las respuestas se agrupan en torno a valores muy cercanos a la media, pero con tendencia elevada.

La variabilidad $\left(C_{V}\right)$ de las respuestas otorgadas a cada competencia analizada se muestra homogénea, exceptuando aquellas relativas al aprendizaje autónomo (V20 $-C_{V}=23,5 \%$ ) y a la comunicación audiovisual $\left(C_{V}=38,3 \%\right)$, en las cuales se observa una menor y mayor dispersión, respectivamente, lo cual indica una menor y mayor heterogeneidad en las respuestas proporcionadas en cada una.

En un análisis más pormenorizado de cada variable, pronto destaca el trabajo en equipo (V21 $-x=3,89$ ) como la competencia mejor puntuada por nuestros encuestados. Le siguen el conocimiento y prevención de los riesgos laborales (V22 $-x=3,79)$ y el aprendizaje autónomo (V20 $-x=3,77)$, lo que permite identificarlas como aquellas con un nivel de desarrollo más elevado.

Como segundo objetivo perseguido en esta investigación se estableció identificar variables que influyen en la autopercepción que poseen los sujetos participantes respecto a su nivel de desarrollo competencial profesional.

Para dar respuesta a este objetivo, se comprobó si existe relación significativa entre las variables que caracterizan a la muestra (sexo, edad, familia profesional de pertenencia, modo de acceso a la FP Básica y deseo de continuación de estudios) y las competencias analizadas (V18 a V31). Dadas las características de las variables manejadas, se ha aplicado la prueba chi-cuadrado de Pearson. 
De este modo, primeramente, se comprobó la existencia de relación significativa entre las competencias y el sexo de la muestra. Los resultados obtenidos muestran condiciones de dependencia entre esta última y el desarrollo de competencias relacionadas con la educación cívica y constitucional, pues el valor de chi-cuadrado $\left(\chi^{2}=10,134\right)$ presenta una significación de $p=0,038$.

Lo mismo sucede con el desarrollo de competencias relacionadas con la comunicación audiovisual, donde se observa una relación significativa entre ambas variables $\left(\chi^{2}=10,341-p=0,035\right)$ y, por tanto, dependencia entre las mismas.

Por otro lado, obtuvimos datos reveladores acerca del grado de asociación significativo entre la edad y el desarrollo de competencias relacionadas con la educación civica y constitucional $\left(\chi^{2}=33,717-p=0,028\right)$, así como con el desarrollo de competencias relacionadas con las TIC $\left(\chi^{2}=36,690-p=0,013\right)$. Estos datos nos permiten descartar la hipótesis de independencia entre estos pares de variables y demostrar su relación de dependencia.

En cuanto a la variable familia profesional se han constatado relaciones estadísticas entre esta y el desarrollo de competencias propias del título de formación profesional $\left(\chi^{2}=54,561-p=0,000\right)$, comprender la organización $y$ las características del sector productivo correspondiente y los mecanismos de inserción profesional $\left(\chi^{2}=66,648-p=0,000\right)$, desarrollo del espiritu emprendedor $\left(\chi^{2}=51,977-p=0,001\right)$, desarrollo de competencias relacionadas con la educación civica y constitucional $\left(\chi^{2}=48,952-p=0,002\right)$, desarrollo de competencias relacionadas con la comunicación audiovisual $\left(\chi^{2}=38,133-p=0,034\right)$ y preparación para la continuación de estudios $\left(\chi^{2}=43,597-p=0,008\right)$. Todos estos datos revelan un fuerte grado de dependencia entre un mayor número de competencias - concretamente seis de ellas - con la familia profesional de los participantes, lo que puede aportar indicios de su mayor influencia en términos estadísticos.

Se ha descartado la hipótesis de dependencia entre la modalidad de acceso a la FP Básica y cada una de las competencias incluidas en el análisis. Esto permite afirmar que no existe relación significativa $y$, por consiguiente, no podemos identificarla como un elemento influyente en la percepción del desarrollo competencial.

Finalmente, se comprobó si existe algún grado de asociación entre el deseo de la muestra participante de continuar con sus estudios al finalizar el ciclo actual y las restantes variables aquí tratadas. Como era esperable, existe correlación entre el deseo de continuar estudiando y la competencia de preparación para la continuación en el sistema educativo, pues el valor $\chi^{2}=10,959$ presenta una significación de $p=0,027$, lo que permite confirmar la hipótesis de relación de variables en condiciones de dependencia. Así mismo, también se observa relación significativa y, por tanto, dependencia en un alto grado, entre el desarrollo de competencias vinculadas con las TIC y la continuación de estudios $\left(\chi^{2}=14,030-p=0,007\right)$. 


\section{Discusión y conclusiones}

Según los resultados de este estudio, se confirma la elevada presencialidad masculina en la FP Básica, siguiendo la tendencia estatal existente (MEFP, 2020) en las enseñanzas de formación profesional. Así mismo, dicha presencialidad supera con creces a la femenina en determinadas familias profesionales como electricidad y electrónica o automoción, en las cuales la existencia de alumnas es muy residual, como ya se había puesto de manifiesto en otros trabajos precedentes (Mariño y Rial, 2018).

Por otro lado, la ESO es la principal vía de acceso a las enseñanzas de FP Básica, siendo interesante observar que un amplio número de participantes no hubiera deseado seguir en la secundaria obligatoria, aunque sí manifiesta intención de continuar con sus estudios una vez finalizado el ciclo actual.

Igualmente, de conformidad con los datos obtenidos, podemos señalar que la FP Básica contribuye en buena medida a la adquisición de competencias para el desarrollo profesional y para la continuidad escolar. En general, los participantes en este estudio consideran tener un desarrollo competencial medio o incluso medio-alto, a la vez que señalan el trabajo en equipo, el conocimiento y prevención de los riesgos laborales y el aprendizaje autónomo como aquellas competencias desarrolladas en mayor grado gracias a la FP Básica. En este sentido, no podemos olvidar que la investigación sobre FP Básica pone de relieve que el rendimiento del alumnado es uno de los elementos que más se asocian con el funcionamiento de los centros de Formación Profesional Básica (Aramendi, Lizasoain y Lukas, 2018). Habría, por tanto, que profundizar en el conocimiento de la dimensión organizativa y detectar aquellos elementos más favorecedores para el desarrollo competencial e intervenir sobre ellos.

Así mismo, se han podido identificar aquellas variables que pueden estar interviniendo en la percepción de la muestra participante con relación al desarrollo competencial. Los resultados evidencian que el género influye en la percepción que el alumnado posee sobre el desarrollo de aquellas competencias que podríamos denominar transversales — relativas a factores como la educación cívica o las TIC_- mientras que la variable familia profesional interviene en el nivel de percepción que los participantes muestran de las competencias más vinculadas con la profesionalización del individuo, como son la adquisición de las propias habilidades de la profesión o la comprensión de las características del sector productivo de pertenencia.

Estos resultados revelan, en la línea de lo que ya indicaban Serrano y Soler (2015), que la FP Básica, entendida como medida inclusiva (Martínez Carmona, 2018), puede convertirse en una vía útil para reducir el abandono temprano y favorecer la inserción laboral, para la prevención del fracaso escolar y el abandono temprano de la educación, así como para la inserción laboral del alumnado, recogiendo el testigo de otras acciones precedentes que iniciaban este camino de «reenganche» educativo y laboral (Amores Fernández y Rittaco Real, 2015; Sarceda-Gorgoso et al., 2017). En este sentido, podemos afirmar que la FP Básica favorece el desarrollo de las primeras competencias 
profesionales del alumnado que, aunque de un nivel de cualificación I, son esenciales para iniciarse en una actividad profesional. Este hallazgo coincide parcialmente con los de estudios previos que ponen el foco de atención desde la perspectiva de los agentes empresariales, quienes valoran positivamente el nivel de competencias básicas del alumnado de FP Básica (Olmos-Rueda y Mas-Torrelló, 2017), destacando especialmente su capacidad de adaptabilidad.

Así pues, es imprescindible promover la continuidad de los jóvenes en el sistema educativo y potenciar el desarrollo de competencias. Este desafío requiere de medidas concretas en varios frentes. Con relación a los estudios de formación profesional, es necesario desarrollar tanto políticas de apoyo que mejoren su atractivo como mecanismos para promover la perseverancia y el éxito de quienes la cursan (Salvà-Mut, Tugores-Ques, Adame-Obrador y Oliver-Trobat, 2017). En lo que respecta al profesorado, se demanda mejorar su capacitación para enfrentarse a las nuevas tareas educativas generadas por la diversidad (Cernadas Ríos, Lorenzo Moledo y Santos Rego, 2019), así como el empleo de metodologías activas con diversidad de estrategias didácticas que se muestran como favorecedoras, en formación profesional, del desarrollo de competencias de índole personal, social y profesional (Campaña-Jiménez, Gallego-Arrufat y Muñoz-Leiva, 2019). Como afirman Brunet et al. (2013), las condiciones laborales dependen de la eficacia del sistema educativo en cuanto a las dimensiones cualitativa y cuantitativa de la formación ofertada, lo cual potenciará o limitará la empleabilidad, especialmente si el foco de atención se pone en los jóvenes más vulnerables.

Así mismo, el éxito de la FP Básica conlleva seguir trabajando en favor de la calidad organizativa y docente de esta etapa (González-González y CutandaLópez, 2020), de la ampliación de la oferta de estos programas, dotándolos de contenidos significativos (Sarceda-Gorgoso, et al., 2017) y de su adaptabilidad a un colectivo de alumnado con un perfil caracterizado por la vulnerabilidad socioeducativa - marcada, entre otros muchos aspectos, por entornos sociofamiliares desfavorecidos (Márquez y Gualda, 2014; Vallejo, 2017) — o por una elevada falta de motivación y de expectativas académicas y laborales (Olmos-Rueda y Mas-Torrelló, 2013; Pérez et al., 2016). En definitiva, se trata de mejorar y repensar la FP Básica en términos de inclusión, concepto que «va ligado al de éxito escolar, y sin una respuesta educativa que tenga en cuenta la heterogeneidad y pluriculturalidad del alumnado, y sus circunstancias comunitarias, no habrá éxito entre los alumnos, sobre todo de aquellos más vulnerables» (Escarbajal Frutos, Essomba Gelabert y Abenza Pastor, 2019, p. 81).

Los hallazgos obtenidos en este trabajo, sin duda, son importantes para aproximarse a la visión que el alumnado de FP Básica tiene sobre su nivel de desarrollo competencial, a la vez que podemos vislumbrar qué variables están influyendo en esta percepción. Sin embargo, a pesar del interés de los datos, el estudio presenta una serie de limitaciones que será necesario superar en el futuro.

Primeramente, cabe destacar el tamaño de la muestra, que debería ser ampliado tanto en número como en lo que respecta a otras comunidades autónomas, dadas las diferencias entre ellas en el éxito académico del alumna- 
do de FP Básica (Sarceda-Gorgoso et al., 2017). Esto permitiría identificar aspectos diferenciales sobre los que actuar para promover la mejora cualitativa de estas enseñanzas.

En segundo lugar, y aunque la dimensión perceptiva es habitual en la investigación educativa, no cabe duda de que no es reflejo de la realidad objetiva, sino de la realidad subjetiva individual, y no podemos olvidar que estas dos dimensiones no siempre van de la mano, tal y como ponen de manifiesto Abiétar-López, Navas-Saurin, Marhuenda-Fluixá y Salvà-Mut (2017), lo que lleva a considerar la oportunidad de servirse de metodologías complementarias que permitan profundizar en la comprensión del problema y eviten una lectura descontextualizada, así como la diversificación de fuentes de información. En este sentido, se considera necesario superar la vertiente exclusivamente cuantitativa y adoptar una metodología mixta que ayude a profundizar en la dimensión contextual del proceso. El método biografíco podría ayudar en esto. Por otra parte, la participación de los agentes económicos permitiría dotar a este estudio de unos informadores clave para constatar el desarrollo efectivo de competencias profesionales, dado que estas solo se muestran en los contextos específicos de trabajo.

Dichas limitaciones son asumidas por la autoría del presente documento y en ello se está trabajando en este momento.

\section{Referencias bibliográficas}

Abiétar-López, M.; Navas-Saurin, A.A.; Marhuenda-Fluixá, F. y Salvà-Mut, F. (2017). La construcción de subjetividades en itinerarios de fracaso escolar: Itinerarios de inserción sociolaboral para adolescentes en riesgo. Psychosocial Intervention, 26(1), 39-45. $<$ http://dx.doi.org/10.1016/j.psi.2016.07.002>

Amores Fernández, J. y Ritacco Real, M. (2015). De los Programas de Cualificación Profesional Inicial (PCPI) como medida de prevención del fracaso escolar a la Formación Profesional Básica: Un estudio sobre el éxito y fracaso de alumnos en riesgo de exclusión educativa en Educación Secundaria. Revista de Investigación en Educación, 13(1), 105-120. Recuperado de <https://revistas.webs.uvigo.es/ index.php/reined/article/view/2046>.

Aramendi, P.; Lizasoain, L. y LuKas, J.F. (2018). Organización y funcionamiento de los centros educativos de Formación Profesional Básica. Bordón, 70(2), 9-23.

Brunet, I.; Pizzi, A. y Valls, F. (2013). Condiciones de vida y construcciones de identidades juveniles: El caso de los jóvenes pobres y excluidos en España. Revista Mexicana de Sociología, 75(4), 647-674. $<$ http://dx.doi.org/10.22201/iis.01882503p.2013.4.42271>

Campaña-Jiménez, R.L.; Gallego-Arrufat, M.J. y Muñoz-Leiva, F. (2019). Estrategias de enseñanza para la adquisición de competencias en formación profesional: Perfiles de estudiantes. Educar, 55(1), 203-229. $<$ https://doi.org/10.5565/rev/educar.876>

Cernadas Ríos, F.; Lorenzo Moledo, M. y Santos Rego, M. (2019). Diversidad cultural y escenarios migratorios: Un estudio sobre formación de profesores. $E d u-$ car, 55(1), 19-37.

<https://doi.org/10.5565/rev/educar.961> 
Deutsche Bank (2020). Evolución bolsa española. Informe de Inversión. 1 al 30 de junio de 2020. Recuperado de <https://www.deutsche-bank.es/pbc/docs/renta_ variable_espana.pdf $>$.

Durán, P. (2005). Los datos perdidos en estudios de investigación ¿`son realmente datos perdidos? Archivos Argentinos de Pediatría, 103(6), 566-568. Recuperado de <https://www.sap.org.ar/publicaciones/archivos/numeros-anteriores/146/Diciembre-2005.html>.

Escarbajal Frutos, A.; Essomba Gelabert, M. y Abenza Pastor, B. (2019). El rendimiento académico de alumnos de la ESO en un contexto vulnerable y multicultural. Educar, 55(1), 79-99. <https://doi.org/10.5565/rev/educar.967>

EURYDICE y CEDEFOP (2014). La lucha contra el abandono temprano de la educación y la formación en Europa: Estrategias, politicas y medidas. Luxemburgo: Oficina de publicaciones de la Unión Europea. <https://doi.org/10.2797/483164>

González-González, M.T. y Cutanda-López, M.T. (2020). Programas de Reenganche educativo y condiciones organizativas para su implementación: La importancia de la coordinación curricular. Educatio Siglo XXI, 38(2) (julio-octubre), 17-44. <https://doi.org/10.6018/educatio.410721>

Fernández-García, A.; García, J.L. y García, M. (2019). La formación profesional básica, una alternativa para atender las necesidades educativas de los jóvenes en riesgo social. Revista de Humanidades, 36, 211-232. <https://doi.org/10.5944/rdh.36.2019.22564>

«Ley Orgánica 5/2002, de 19 de junio, de las Cualificaciones y de la Formación Profesional». Boletin Oficial del Estado, 147 (20 de junio).

«Ley Orgánica 8/2013, de 9 de diciembre, para la Mejora de la Calidad Educativa». Boletin Oficial del Estado, 295 (10 de diciembre).

Mariño, R. y Rial, A. (2018). Balance de las necesidades formativas demandadas por mujeres que cursan o que han cursado ciclos de FP en familias profesionales masculinizadas: El caso de Galicia. Educar, 55(1), 251-272. $<$ https://doi.org/10.5565/rev/educar.890>

Márquez, C. y Gualda, E. (2014). Absentismo escolar en Secundaria: Diferencias entre nacionales e inmigrantes en la provincia de Huelva. En-clave Pedagógica: Revista Internacional de Investigación e Innovación Educativa, 13, 55-66. Recuperado de <http://www.uhu.es/publicaciones/ojs/index.php/xxi/article/view/2093>.

Martínez Carmona, M.J. (2018). Análisis y valoración de los ciclos de Formación Profesional Básica como medida de atención a la diversidad a través del alumnado y del profesorado. Universidad de Córdoba. Tesis doctoral. Recuperado de <https:// helvia.uco.es/bitstream/handle/10396/16402/2018000001752.pdf?sequence= 1yisAllowed $=\mathrm{y}>$.

MEFP (2020). Igualdad en cifras MEFP 2020: Aulas por la igualdad. Madrid: Ministerio de Educación y Formación Profesional. Recuperado de <https://www.edu cacionyfp.gob.es/dam/jcr:914e956e-9241-49c5-b9a6-d99d6eade751/igualdaden-cifras-2020-online.pdf>.

Montenegro, E.; Oh, Y. y Chesnut, S. (2015). No le tema a los datos perdidos: Enfoques modernos para el manejo de datos perdidos. Actualidades en Psicología, 29(119), 29-42. <https://doi.org/10.15517/ap.v29i119.18812> 
Moreno-Guerrero, A.J.; Fuentes Cabrera, A. y López Belmonte, J. (2019). Las competencias digitales del alumnado de Formación Profesional Básica. Revista de Educación de la Universidad de Granada, 26, 9-33. $<$ http://doi.org/10.30827/reugra.v26i0.111>

Moreno-Guerrero, A.J.; López, J.; Pozo, S. y Fuentes, A. (2019). Influencia del contexto en el uso de dispositivos TIC en la Formación Profesional Básica. EDMETIC: Revista de Educación Mediática y TIC, 9(1), 149-169. $<$ https://doi.org/10.21071/edmetic.v9i1.12195>

Negri, M.I. y Leiva, J.J. (2019). Liderazgo de los docentes de formación profesional básica para la mediación escuela-empleo del alumnado con diversidad funcional intelectual. Contextos Educativos, 24, 95-111. <http://doi.org/10.18172/con.3902>

OECD (2016a). Education at a Glance 2016: OECD Indicators. París: OECD Publishing. <http://dx.doi.org/10.1787/eag-2016-en>

- (2016b). Estudiantes de bajo rendimiento: Por qué se quedan atrás y cómo ayudarles a tener éxito. París: OECD Publishing. Recuperado de <http://www.oecd.org/pisa/ keyfindings/PISA-2012-Estudiantes-de-bajo-rendimiento.pdf $>$.

Olmos-Rueda, P. y Mas-Torelló, O. (2013). Jóvenes, fracaso escolar y programas de segunda oportunidad. Revista Española de Orientación y Psicopedagogía, 24(1), 78-93. $<$ https://doi.org/10.5944/reop.vol.24.num.1.2013.11272>

- (2017). Perspectiva de tutores y de empresas sobre el desarrollo de las competencias básicas de empleabilidad en el marco de los programas de formación profesional básica. Educar, 53(2), 261-284. $<$ https://doi.org/10.5565/rev/educar.870>

Pérez, V.; Velázquez, M.; Villanueva, A.; Sánchez, L.M. y Fernández, M.C. (2016). Percepción y significación de jóvenes excluidos del contexto escolar o laboral y consumo de sustancias psicoactivas. Health and Addictions: Saludy Drogas, 16(1), 19-32. <http://dx.doi.org/10.21134/haaj.v16i1.248>

«Real Decreto 127/2014, de 28 de febrero, por el que se regulan aspectos específicos de la Formación Profesional Básica». Boletín Oficial del Estado, 55 (5 de marzo).

Salvà-Mut, F.; Tugores-Ques, M.; Adame-Obrador, M. y Oliver-Trobat, M. (2017). La inserción laboral de los jóvenes y las jóvenes con titulación de formación profesional de grado medio y superior en España. Educar, 53(2), 357-378. <https://doi.org/10.5565/rev/educar.869>

Sarceda-Gorgoso, M.C.; Santos-González, M.C. y Sanjuán Roca, M.M. (2017). La Formación Profesional Básica: ¿alternativa al fracaso escolar? Revista de Educación, 378, 78-112. <https://doi.org/10.4438/1988-592X-RE-2017-378-362>

Sarceda-Gorgoso, M.C.; Santos-GonzÁlez, M.C.; Barreira-Cerqueiras, E.M. y Sanjuán-Roca, M.M. (2019). Atención a la diversidad, éxito educativo y empleabilidad: De los programas de garantía social a la formación profesional básica. En Miguel Ángel Santos Rego, Antonio Valle Arias y Mar Lorenzo Moledo (eds.). Exito educativo: Claves de construcción y desarrollo (pp. 255-272). Valencia: Tirant Lo Blanch.

Serrano, L. y Soler, A. (2015). La formación y el empleo de los jóvenes españoles: Trayectoria reciente y escenarios futuros. Bilbao: Fundación BBVA. Recuperado de 
<https://www.fbbva.es/wp-content/uploads/2017/05/dat/DE_2015_formacion_ y_empleo.pdf $>$.

Tejada, J. (1999). Acerca de las competencias profesionales I. Revista Herramientas, 56, 20-30.

VAlLejo, A. (2017). La generación Y ante el desafío de su inserción laboral: Realidades frente a estereotipos. Arbor: Ciencia, Pensamiento y Cultura, 193(786), 1-14. $<$ https://doi.org/10.3989/arbor.2017.783n1006> 